\title{
Evaluación financiera de la producción y venta de madera aserrada de Eucalyptus globulus Labill elaborada con sierra circular y de cinta
}

\begin{abstract}
Financial evaluation of the production and sale of Eucalyptus globulus Labill sawn wood made with a circular and band saw
\end{abstract}

\author{
Miguel Ángel Guallpa Calva. ${ }^{1}$, José Franklin Arcos Torres. ${ }^{2}$, José Pedro Suatunce \\ Cunuhay. ${ }^{3} \&$ Hayron Fabricio Canchignia Martínez. ${ }^{4}$
}

\section{DOI: $\underline{\text { https://doi.org/10.33262/visionariodigital.v5i1.1541 }}$}

\begin{abstract}
.
Introduction. The need to know the economic situation of two production units of sawn wood, which have a production capacity already installed, motivated to develop this financial assessment of the performance in the process of transformation of round wood to square wood of Eucalyptus globulus, with the use of circular saw (sawmill A1) and circular saw plus band saw (sawmill A2). Objectives. Establish production costs and profitability for the sale of first, second and coastal boards based on the sawing systems used. Methodology. In this research observation and interview techniques were applied. The costs were estimated by grouping the components; production, administration, sales and financial level. The profitability with the calculation of gross profit margin (MBU) and net profit margin (MUN). Results. The results show similar production costs when preparing first, second and coastal tables of E. globulus with values of 0.29 and 0.27 USD / pt in sawmills A1 and A2

\footnotetext{
${ }^{1}$ Escuela Superior Politécnica de Chimborazo, Chimborazo-Riobamba, miguel.guallpa@espoch.edu.ec https://orcid.org/0000-0001-5392-036X

${ }^{2}$ Escuela Superior Politécnica de Chimborazo, Chimborazo-Riobamba, jarcos@espoch.edu.ec https://orcid.org/0000-0002-6465-3751

${ }^{3}$ Universidad Técnica Estatal de Quevedo, Los Ríos-Quevedo, jsuatunce@uteq.edu.ec https://orcid.org/00000002-9578-923X

${ }^{4}$ Universidad Técnica Estatal de Quevedo, Los Ríos-Quevedo, hcanchignia@uteq.edu.ec https://orcid.org/0000-0003-1195-5446
} 
respectively. On the other hand, with the transaction of the three wood products, they generate a gross profit of $53.79 \%, 61.49 \%$ and as a net profit margin of 61 and 87 dollars respectively. Conclusion. The profitability of the two sawmill projects for selling first-class, second-class and coastal Eucalyptus boards generated gross profit margin values higher than $50 \%$, ideal for these businesses, and in the case of the sawmill A2 net profit margin As it is the highest at 26 USD, it is the best in reference to the A1 sawmill.

Keywords: Sawmill, wood, production costs, sale, financial profit

\section{Resumen}

Introducción. La necesidad de conocer la situación económica de dos unidades productoras de madera aserrada, que cuentan con una capacidad productiva ya instalada, motivó a desarrollar esta valoración financiera del rendimiento en el proceso de transformación de madera en rollo a madera escuadrada de Eucalyptus globulus, con el uso de sierra circular (aserradero A1) y sierra circular más sierra de cinta (aserradero A2). Objetivos. Establecer los costos de producción y la rentabilidad por la venta de tablas de primera, tablas de segunda y costeras con base en los sistemas de aserrío utilizados. Metodología. En esta investigación se aplicó las técnicas de observación y entrevista. Los costos se estimaron al agrupar los componentes; producción, administración, el nivel de ventas y financiero. La rentabilidad con el cálculo del margen bruto de utilidad (MBU) y margen de utilidad neta (MUN). Resultados. Los resultados evidencian costos de producción similares al momento de elaborar tablas de primera, tablas de segunda y costeras de E. globulus con valores de 0,29 y 0,27 USD/pt en los aserraderos A1 y A2 respectivamente. Por su parte, con la transacción de los tres productos de madera, generan utilidad bruta de 53,79\%, 61,49\% y como margen de utilidad neta 61 y 87 dólares respectivamente. Conclusión La rentabilidad de los dos proyectos de aserradero por vender tablas de primera, tablas de segunda y costeras de eucalipto generaron valores de margen de utilidad bruta superiores al 50\%, idóneos para estos negocios, y en el caso del margen de utilidad neta del aserradero A2 por ser más alto con 26 USD, es el mejor en referencia al aserradero A1.

Palabras claves: Aserrío, madera, costos de producción, venta, rentabilidad financiera

\section{Introducción.}

La industria maderera en Ecuador es fundamental para el desarrollo del país, por la creación de fuentes de trabajo, producción de bienes y servicios (Ministerio del Ambiente del Ecuador. MAE, 2013), su configuración está integrada por las fases de silvicultura e industrialización para la producción de los diversos productos forestales, siendo una de las especies más empleadas para la elaboración de madera aserrada el Eucalyptus globulus procedente de bosques plantados en nuestro país (López \& Muñoz, 2017), ocupando el primer lugar con el $19,43 \%$ del volumen total de madera aprovechada, cuya industrialización está compuesta por 
la transformación primaria y secundaria (MAE, 2010; Añazco et al., 2010), en la fase primaria de producción de madera aserrada en Ecuador en 2011 con $519 \mathrm{mil} \mathrm{m}^{3}$, por ello constituye el producto con el mayor crecimiento con $11 \%$ en relación al año 2010 (PRO ECUADOR, 2013).

Para la región sierra, en el caso de aserraderos, una investigación indica altos consumos de residuos madereros de eucalipto con valores entre el 34-41.03\% (Guallpa et al., 2018), otro estudio de productividad del aserrío de madera en dos fábricas en Riobamba, reportó con el uso de sierra circular en el establecimiento A el 27,74 \% y en B, provisto de sierra circular y otra de cinta con el 48,84\% de productividad respectivamente (Guallpa, Suatunce \& Canchignia, 2019). La industria maderera en Riobamba, dispone actualmente de cierta información técnica de productividad de madera aserrada en E. globulus, no así de la rentabilidad, siendo muy necesaria para generar estrategias de gestión de la madera escuadrada en aserradero, en este caso por la elaboración, y transacción de tablas o costeras de eucalipto producidas con sierra circular y de cinta.

Según el criterio de Ecuador forestal (2013), el tipo de aserrío determina el nivel de aprovechamiento de la materia prima usada para la obtención de tablas, tablones, vigas entre otros, para su conversión, utiliza maquinaria, equipo, recurso humano, fuentes de energía y dinero; durante un tiempo y derivado de un diferente nivel tecnológico, lo cual incide en la productividad de la transformación primaria y que repercute en los costos de producción (García et al., 2001).

Al diferenciar los costos y rentabilidad que implica la organización de su producción, cuyo manejo adecuado permitirá obtener mejoras en todo el proceso de transformación de acuerdo con lo expresado por Maturana, Pizani \& Vera. (2010). Según su nivel tecnológico que poseen cada fábrica, constituyen los elementos que sustentaron el desarrollo de esta investigación, cuyos resultados permitirán la toma de decisiones de manejo eficiente de las operaciones de producción para que se eleve el grado de productividad con la consecuente disminución de residuos forestales, cuyo fin pretende evidenciar una mayor rentabilidad por la producción de madera aserrada en beneficio de desarrollo forestal del país (Secretaría Nacional de Planificación y Desarrollo, 2017).

Para ello, es necesario calcular ciertos indicadores de rentabilidad requeridos para la gestión de los administradores en la ejecución de sus funciones inherentes, debido a que permiten evaluar el cumplimiento de las actividades planificadas con las metas alcanzadas y de esta manera determinar, si la administración aplicando las estrategias adecuadas, se enfocan a lograr un crecimiento de las fábricas (Fontalvo, Mendoza \& Morelos, 2011); los objetivos del presente estudio fueron: Establecer los costos de producción y la rentabilidad por la venta de tablas de primera, tablas de segunda y costeras de Eucalyptus globulus en función del sistema de aserrío utilizado. 


\section{Metodologia.}

Por las particularidades de las instalaciones de 12 aserríos del cantón Riobamba, se agruparon en 4 con sierra circular y 8 restantes conformados por sierra circular y de cinta como población de referencia, se optó por la selección de dos aserraderos, el primero se designa A1, está provisto de una sierra circular y el segundo se le llama A2, el cual tiene una sierra circular más otra de cinta.

Las dos fábricas son de propiedad particular dedicadas a la elaboración y comercialización de madera aserrada de E. globulus como tablas y costeras. Ambas instalaciones están dentro del cantón Riobamba. El aserradero A1 por su parte, se encuentra ubicado a $5 \mathrm{~km}$ de la vía Riobamba-Quimiag cerca al desvío a San Gerardo y el aserradero A2 se localiza a $200 \mathrm{~m}$ del mercado mayorista de la ciudad de Riobamba (Guallpa, Suatunce \& Canchignia, 2019).

Tabla 1. Localización geográfica de los aserraderos del estudio

\begin{tabular}{cccc}
\hline Aserraderos & X & Y & Altitud (msnm) \\
\hline A1 & 764353 & 9817384 & 2750 \\
A2 & 763654 & 9813400 & 2745 \\
\hline
\end{tabular}

Fuente: (Guallpa, 2018)

\section{Métodos}

En esta investigación, se aplicaron los métodos: documental, de campo y explicativa. Dentro de las técnicas se utilizó la observación directa y entrevistas sobre la base de un cuestionario aplicado a los actores claves tanto a propietarios, obreros y expertos con el objeto de diagnosticar la situación actual de los dos aserríos en lo referente a materia prima, materiales e insumos, personal, el consumo de combustible, energía eléctrica, mantenimiento, el volumen de madera y precio de venta de los tres productos derivados de la madera de $E$. globulus, la parte de gestión administrativa y financiera de cada aserradero (García de la Figal, 2018).

La recopilación de la información de costos de producción y el análisis de rentabilidad del estudio tuvo lugar desde enero a mayo del año 2018.

\section{Determinación de los costos y rentabilidad del aserrado}


Con base en los datos de costos, gastos e ingresos por las ventas de tres tipos de productos de madera de eucalipto indicados, más la incorporación de elementos como la inversión en activos fijos, diferidos, el capital de trabajo, amortización de la deuda, la depreciación en activos fijos y diferidos, se elaboró el presupuesto de costos y gastos de cada aserradero (Cubbage, Davis \& Frey, 2011). La información recopilada, se utilizó en la elaboración de los estados de resultados para un periodo de 5 meses (Muñoz, 2010). Finalmente, se calculó el Margen bruto de utilidad (MBU) y Margen de utilidad neta (MUN) según esquema de cálculo propuesto por Hidalgo \& Jaramillo (2009).

$$
\mathrm{MBU}=\frac{\mathrm{UB}}{\mathrm{VN}} * 100
$$

Donde:

MBU = Margen bruto de utilidad

$\mathrm{UB}=$ Utilidad bruta

$\mathrm{VN}=$ Ventas netas

$$
\mathrm{MUN}=\frac{\mathrm{UN}}{\mathrm{Cts}} * 100
$$

Donde:

MNU = Margen de utilidad neta

UN = Utilidad neta

Cts $=$ Costos

\section{Cálculo de indicadores}

Para el cálculo de indicadores financieros se usó la gestión de datos de la hoja de cálculo de Excel elaborada por Pacheco (2015).

\section{Resultados y discusión}

\section{Costos de producción}

Para el aserradero A1, se obtuvo un costo 0,29 USD por pt de eucalipto, cuyo valor demuestra la mayor incidencia de materia prima con el 44,28\%, seguido de la gestión administrativa con el $23,48 \%$, y el componente mano de obra con $13,26 \%$, en cambio la menor incidencia corresponde a la depreciación de elementos que conforman la depreciación del componente administrativo con el $0,44 \%$ al trabajar con sierra circular y se determinó un costo total ligeramente inferior de 0,272 USD por pt al manejar el sistema sierra circular + sierra de cinta del aserradero A2 (Tabla 2). 
Tabla 2. Costos variables y fijos de la producción de los aserraderos evaluados

\begin{tabular}{|c|c|c|c|c|c|c|}
\hline \multirow{3}{*}{ Clasificación } & \multirow{3}{*}{ Área } & \multirow{3}{*}{ Elementos } & \multicolumn{4}{|c|}{ Aserraderos } \\
\hline & & & \multicolumn{2}{|c|}{$\mathrm{A} 1$} & \multicolumn{2}{|c|}{$\mathrm{A} 2$} \\
\hline & & & $\begin{array}{c}\text { Costo } \\
\text { (USD/pt) }\end{array}$ & $(\%)$ & $\begin{array}{l}\text { Costo } \\
\text { (USD/pt) }\end{array}$ & $(\%)$ \\
\hline \multirow{2}{*}{$\begin{array}{l}\text { Costo } \\
\text { variable }\end{array}$} & \multirow{2}{*}{ Producción } & Materia prima & 0,1282 & 44,28 & 0,076 & 27,84 \\
\hline & & $\begin{array}{l}\text { Combustible y } \\
\text { energía eléctrica }\end{array}$ & 0,002 & 0,82 & 0,015 & 5,51 \\
\hline \multirow{10}{*}{ Costo fijo } & \multirow{5}{*}{ Producción } & Arrendamiento & 0,004 & 1,44 & 0,004 & 1,47 \\
\hline & & Mano de obra & 0,038 & 13,26 & 0,054 & 19,83 \\
\hline & & $\begin{array}{l}\text { Herramientas y } \\
\text { materiales }\end{array}$ & 0,022 & 7,47 & 0,02 & 7,34 \\
\hline & & Mantenimiento & 0,013 & 4,42 & 0,017 & 6,24 \\
\hline & & Depreciación & 0,003 & 0,87 & 0,005 & 1,84 \\
\hline & \multirow{3}{*}{ Administración } & Administración & 0,068 & 23,48 & 0,05851 & 21,49 \\
\hline & & & & & & \\
\hline & & Depreciación & 0,001 & 0,44 & 0,001 & 0,37 \\
\hline & Ventas & Ventas & 0,003 & 1,04 & 0,009 & 3,31 \\
\hline & Financiero & Financiero & 0,007 & 2,49 & 0,013 & 4,77 \\
\hline & & Total & 0,290 & 100,00 & 0,272 & 100,00 \\
\hline
\end{tabular}

Fuente: (Guallpa, 2018)

Así mismo para el aserradero A2 se determinó un menor efecto que ejerce la materia prima con el $27,84 \%$, le sigue la parte administrativa con el $21,49 \%$, la mano de obra con 19,83\% y con menor influencia el valor de depreciación del componente administración con el 0,37\% (Hernández, 2016). Los costos de producción por pie tablar proyectados en el presente estudio son similares al reportado por (Coronel de Renolfi et al., 2012) quien determinó un costo de aserrío de USD \$ 0.31 para Prosopis alba Griseb en Santiago del Estero, Argentina, dicho costo fue tipificado basándose en los rubros de materia prima, mano de obra directa y energía consumida en el proceso de aserrío, al relacionar con el tipo de cambio de julio de 2018, equivaldría a USD \$1.47, siendo menores los costos de producción por pie tablar estimados en los dos aserraderos del cantón Riobamba con alrededor del $79 \%$. 


\section{Rentabilidad financiera}

De acuerdo a los niveles de producción y las ventas registradas, se agrupó los costos, gastos, e ingresos, en la elaboración de los estados de resultados considerando un periodo de 5 meses en cada aserradero, componentes que se muestran a continuación.

\section{Agrupación de costos y gastos del aserradero A1}

El costo total del proceso de aserrado de madera para 50.000 pie tablares que configura a los elementos de producción, administración, las ventas y financiero varió en los cinco meses de valoración en el aserradero A1(Tabla 2).

Tabla 2. Resumen de la agrupación de costos y gastos del aserradero A1 en USD

\begin{tabular}{llllll}
\hline \multirow{2}{*}{ Denominación } & \multicolumn{5}{c}{ Meses 2018 } \\
\cline { 2 - 6 } & \multicolumn{1}{c}{ M1 } & \multicolumn{1}{c}{ M2 } & \multicolumn{1}{c}{ M3 } & \multicolumn{1}{c}{ M4 } & \multicolumn{1}{c}{ M5 } \\
\hline Costos de Producción & $\mathbf{1 0 . 9 6 9 , 7 4}$ & $\mathbf{1 0 . 9 5 8 , 5 1}$ & $\mathbf{1 0 . 9 4 8 , 0 7}$ & $\mathbf{1 0 . 7 2 8 , 6 3}$ & $\mathbf{1 0 . 9 4 8 , 0 7}$ \\
Mano de obra directa & $1.920,00$ & $1.941,63$ & $1.939,66$ & $1.898,37$ & $1.939,66$ \\
Mano de obra indirecta & 480,00 & 485,41 & 484,92 & 474,59 & 484,92 \\
Materia prima, insumos y materiales & $7.725,57$ & $7.812,60$ & $7.804,69$ & $7.638,55$ & $7.804,69$ \\
Combustibles & 78,00 & 78,88 & 78,80 & 77,12 & 78,80 \\
Mantenimiento & 640,00 & 640,00 & 640,00 & 640,00 & 640,00 \\
Depreciación & 126,17 & 126,17 & 126,17 & 126,17 & 126,17 \\
\hline Gastos Administrativos & $\mathbf{3 3 9 9 , 6 1}$ & $\mathbf{3 3 2 4 , 6 8}$ & $\mathbf{3 3 2 1 , 0 8}$ & $\mathbf{3 2 6 9 , 4 8}$ & $\mathbf{3 3 2 1 , 0 8}$ \\
\hline Sueldos y salarios & 246,53 & 120,00 & 120,00 & 120,00 & 120,00 \\
Servicios básicos & 180,00 & 180,00 & 180,00 & 180,00 & 180,00 \\
Materiales de oficina & 21,83 & 21,83 & 21,83 & 21,83 & 21,83 \\
Permisos funcionamiento & 62,50 & 62,50 & 62,50 & 62,50 & 62,50 \\
Depreciación & 177,34 & 177,34 & 177,34 & 177,34 & 177,34 \\
Impuestos por venta de productos & $2.711,40$ & $2.763,00$ & $2.759,40$ & $2.707,80$ & $2.759,40$ \\
\hline Gastos en Ventas & $\mathbf{1 5 0 , 0 0}$ & $\mathbf{1 5 0 , 0 0}$ & $\mathbf{1 5 0 , 0 0}$ & $\mathbf{1 5 0 , 0 0}$ & $\mathbf{1 5 0 , 0 0}$ \\
\hline Promoción y Publicidad & 150,00 & 150,00 & 150,00 & 150,00 & 150,00 \\
Gastos Financieros & $\mathbf{3 6 0 , 7 2}$ & $\mathbf{3 6 0 , 7 2}$ & $\mathbf{3 6 0 , 7 2}$ & $\mathbf{3 6 0 , 7 2}$ & $\mathbf{3 6 0 , 7 2}$ \\
Interés & 360,72 & 360,72 & 360,72 & 360,72 & 360,72 \\
\hline TOTAL & $\mathbf{1 4 . 8 8 0 , 0 7}$ & $\mathbf{1 4 . 7 9 3 , 9 1}$ & $\mathbf{1 4 . 7 7 9 , 8 6}$ & $\mathbf{1 4 . 5 0 8 , 8 3}$ & $\mathbf{1 4 . 7 7 9 , 8 6}$ \\
\hline & & & & & \\
\hline & & & & & \\
& & & & &
\end{tabular}

Fuente: (Guallpa, 2018)

\section{Agrupación de ingresos del aserradero A1}

Los ingresos para el aserradero A1 proviene de la venta de 39.653 pie tablares de eucalipto de primera, 10.108 de segunda y 5.530 costeras a los precios de $0.46,0.25$ y 0.40 USD 
respectivamente en el caso del primer mes y de forma similar para los cuatro meses restantes (Tabla 3).

Tabla 3. Ingresos por la venta de tablas y costeras del aserradero A1

\begin{tabular}{|c|c|c|c|c|c|}
\hline Descripción & M1 & M2 & M3 & M4 & M5 \\
\hline Tablas de primera & 10.200 & 10.400 & 10.200 & 10.000 & 10.200 \\
\hline Pie tablares de primera & 39.653 & 40.431 & 39.653 & 38.876 & 39.653 \\
\hline Precio & 0,46 & 0,46 & 0,46 & 0,46 & 0,46 \\
\hline Subtotal & $18.240,43$ & $18.598,09$ & $18.240,43$ & $17.882,78$ & $18.240,43$ \\
\hline Tablas de segunda & 2.600 & 2.800 & 3.000 & 2.800 & 3.000 \\
\hline Pie tablares de segunda & 10.108 & 10.885 & 11.663 & 10.885 & 11.663 \\
\hline Precio & 0,25 & 0,25 & 0,25 & 0,25 & 0,25 \\
\hline Subtotal & $2.526,91$ & $2.721,29$ & $2.915,67$ & $2.721,29$ & $2.915,67$ \\
\hline Costeras & 6.530 & 6.550 & 6.530 & 6.510 & 6.530 \\
\hline Precio & 0,40 & 0,40 & 0,40 & 0,40 & 0,40 \\
\hline Subtotal & $2.612,00$ & $2.620,00$ & $2.612,00$ & $2.604,00$ & $2.612,00$ \\
\hline Total & 23.379,34 & 23.939,38 & $23.768,10$ & 23.208,07 & $23.768,10$ \\
\hline
\end{tabular}

Fuente: (Guallpa, 2018)

Estado de resultados del aserradero A1

La comparación de las ventas contra los costos, gastos e impuestos del aserradero A1, generaron una utilidad neta de 6.374.46 USD correspondiente al primer mes y de $6.741,18$ USD en el quinto mes (Tabla 4).

Tabla 4. Estado de resultados del aserradero A1

\begin{tabular}{lrrrrr}
\hline \multirow{2}{*}{\multicolumn{1}{c}{ Denominación }} & \multicolumn{6}{c}{ Meses 2018 } \\
\cline { 2 - 6 } (+) Ventas & \multicolumn{1}{c}{ M1 } & \multicolumn{1}{c}{ M2 } & \multicolumn{1}{c}{ M3 } & \multicolumn{1}{c}{ M4 } & \multicolumn{1}{c}{ M5 } \\
\cline { 2 - 6 } (-) Costos de producción & $23.379,34$ & $23.939,38$ & $23.768,10$ & $23.208,07$ & $23.768,10$ \\
\hline (=) Utilidad bruta & $10.969,74$ & $10.958,51$ & $10.948,07$ & $10.728,63$ & $10.948,07$ \\
\hline (-) Gastos administrativos & $12.409,60$ & $12.980,86$ & $12.820,03$ & $12.479,43$ & $12.820,03$ \\
\hline (-) Gastos en ventas & 3399,61 & 3324,68 & 3321,08 & 3269,48 & 3321,08 \\
(=) Utilidad operativa & 150,00 & 150,00 & 150,00 & 150,00 & 150,00 \\
\hline (-) Gastos financieros & 8860,00 & 9506,19 & 9348,96 & 9059,96 & 9348,96 \\
\hline (=) Utilidad antes de impuestos & 360,72 & 360,72 & 360,72 & 360,72 & 360,72 \\
\hline (-) Impuestos 25\% & 8499,28 & 9145,47 & 8988,24 & 8699,24 & 8988,24 \\
(=) Utilidad neta & $2.124,82$ & $2.286,37$ & $2.247,06$ & $2.174,81$ & $2.247,06$ \\
\hline
\end{tabular}

Fuente: (Guallpa, 2018)

Indicadores de rentabilidad del aserradero A1 
Las ventas de la fábrica por comercializar tablas y costeras de eucalipto generaron 53,79\% de utilidad bruta al aserradero A1 para el periodo de 5 meses del año 2018 (Tabla 5).

Tabla 5. Indicadores de rentabilidad del aserradero A1

\begin{tabular}{lc}
\hline \multicolumn{1}{c}{ Denominación } & Total \\
\hline (+) Ventas & $118.062,99$ \\
(-) Costos de producción & $54.553,02$ \\
(=) Utilidad bruta & $63.509,97$ \\
(=) Utilidad neta & $\mathbf{3 3 . 2 4 0 , 3 4}$ \\
& \\
Margen Bruto Utilidad (MBU) & $\mathbf{5 3 , 7 9} \%$ \\
Margen de Utilidad Neta (MUN) & $\mathbf{6 1} \%$ \\
\hline
\end{tabular}

Fuente: (Guallpa, 2018)

El margen de utilidad neta, demuestra que por cada USD 100 que ingresan al negocio, después de pagar los costos (fijos, variables, gastos e impuestos) por elaborar madera aserrada de E. globulus, le sobra USD 61 al aserradero A1 (Tabla 5).

\section{Agrupación de costos y gastos del aserradero A2}

El costo total del proceso de aserrado de madera para 53.000 pie tablares que configura a los elementos de producción, administración, las ventas y financiero mostró un proceder más o menos similar en los cinco meses de valoración en el aserradero A2 del año 2018 (Tabla 6).

Tabla 6. Resumen de la agrupación de costos y gastos del aserradero A2

\begin{tabular}{|c|c|c|c|c|c|}
\hline \multirow{2}{*}{ Denominación } & \multicolumn{5}{|c|}{ Meses 2018} \\
\hline & M1 & M2 & M3 & M4 & M5 \\
\hline Costos de producción & $10.052,02$ & $9.888,70$ & $9.879,58$ & $9.688,00$ & $9.879,58$ \\
\hline Mano de obra directa & $2.400,00$ & $2.427,04$ & $2.424,58$ & $2.372,96$ & $2.424,58$ \\
\hline Mano de obra indirecta & 480,00 & 485,41 & 484,92 & 474,59 & 484,92 \\
\hline Materia prima, insumos y materiales & $5.950,35$ & $6.017,38$ & $6.011,29$ & $5.883,32$ & $6.011,29$ \\
\hline Combustibles & 78,00 & 78,88 & 78,80 & 77,12 & 78,80 \\
\hline Mantenimiento & 880,00 & 880,00 & 880,00 & 880,00 & 880,00 \\
\hline Depreciación & 263,67 & 263,67 & 263,67 & 263,67 & 263,67 \\
\hline Gastos administrativos & 3172,47 & 3101,14 & 3093,94 & 3038,74 & 3086,74 \\
\hline Sueldos y salarios & 246,53 & 120,00 & 120,00 & 120,00 & 120,00 \\
\hline Servicios básicos & 180,00 & 180,00 & 180,00 & 180,00 & 180,00 \\
\hline Materiales de oficina & 21,83 & 21,83 & 21,83 & 21,83 & 21,83 \\
\hline Permisos funcionamiento & 62,50 & 62,50 & 62,50 & 62,50 & 62,50 \\
\hline Depreciación & 63,61 & 63,61 & 63,61 & 63,61 & 63,61 \\
\hline
\end{tabular}


ISSN: 2602-8506

www.visionariodigital.org

Vol. 5, N¹, p. 70-84, Enero-Marzo, 2021

\begin{tabular}{lrrrrr}
\hline Impuestos por venta de productos & $2.598,00$ & $2.653,20$ & $2.646,00$ & $2.590,80$ & $2.638,80$ \\
\hline Gastos en ventas & $\mathbf{5 0 0 , 0 0}$ & $\mathbf{5 0 0 , 0 0}$ & $\mathbf{5 0 0 , 0 0}$ & $\mathbf{5 0 0 , 0 0}$ & $\mathbf{5 0 0 , 0 0}$ \\
\hline Promoción y publicidad & 500,00 & 340,00 & 340,00 & 340,00 & 340,00 \\
\hline Gastos financieros & $\mathbf{7 1 3 , 1 6}$ & $\mathbf{7 1 3 , 1 6}$ & $\mathbf{7 1 3 , 1 6}$ & $\mathbf{7 1 3 , 1 6}$ & $\mathbf{7 1 3 , 1 6}$ \\
\hline Interés & 713,16 & 713,16 & 713,16 & 713,16 & 713,16 \\
\hline TOTAL & $\mathbf{1 4 . 4 3 7 , 6 5}$ & $\mathbf{1 4 . 2 0 3 , 0 0}$ & $\mathbf{1 4 . 1 8 6 , 6 8}$ & $\mathbf{1 3 . 9 3 9 , 9 0}$ & $\mathbf{1 4 . 1 7 9 , 4 8}$ \\
\hline
\end{tabular}

Fuente: (Guallpa, 2018)

\section{Agrupación de ingresos del aserradero A2}

Los ingresos para el aserradero A2 proviene de la venta de 44.070 pie tablares de eucalipto de primera, 11.233 de segunda y 5.800 costeras a los precios de $0.46,0.25$ y 0.40 USD respectivamente en el caso del primer mes y de forma análoga para los cuatro meses restantes (Tabla 7).

Tabla 7. Ingresos por la venta de tablas del aserradero A2

\begin{tabular}{|c|c|c|c|c|c|}
\hline Descripción & M1 & M2 & M3 & M4 & M5 \\
\hline Tablas de primera & 9.600 & 9.800 & 9.600 & 9.400 & 9.200 \\
\hline Pie tablares de primera & 44.070 & 44.934 & 44.070 & 43.205 & 44.070 \\
\hline Precio & 0,46 & 0,46 & 0,46 & 0,46 & 0,46 \\
\hline Subtotal & $20.271,98$ & $20.669,47$ & $20.271,98$ & $19.874,49$ & $20.271,98$ \\
\hline Tablas de segunda & 1.800 & 1600 & 2200 & 2000 & 1800 \\
\hline Pie tablares de segunda & 11.233 & 12.098 & 12.962 & 12.098 & 12.962 \\
\hline Precio & 0,25 & 0,25 & 0,25 & 0,25 & 0,25 \\
\hline Subtotal & $2.808,35$ & 3.024,38 & $3.240,41$ & 3.024,38 & $3.240,41$ \\
\hline Costeras & 5.800 & 5.900 & 5.800 & 5.700 & 5.650 \\
\hline Precio & 0,40 & 0,40 & 0,40 & 0,40 & 0,40 \\
\hline Subtotal & $2.320,00$ & $2.360,00$ & $2.320,00$ & $2.280,00$ & $2.260,00$ \\
\hline Total & $25.400,34$ & $26.053,85$ & $25.832,39$ & $25.178,87$ & $25.772,39$ \\
\hline
\end{tabular}

Fuente: (Guallpa, 2018)

El mejor nivel de ingresos en el aserradero A2, es el resultado del mayor rendimiento obtenido al usar el sistema formado por la sierra circular + sierra de cinta, según el reporte del estudio realizado por Guallpa, Suatunce \& Canchignia. (2019), cuya combinación de equipos y esquema de producción permite disminuir la acumulación de los residuos, se mejora el proceso productivo al optimizar la materia prima y generar mayores ingresos económicos (Soto et al., 2000).

Estado de resultados del aserradero A2 
La confrontación de las ventas contra los costos, gastos e impuestos del aserradero A2, generaron una utilidad neta de 8.222,01 USD correspondiente al primer mes y de 8.694,68 USD en el quinto mes (Tabla 8).

Tabla 8. Estado de resultados del aserradero A2

\begin{tabular}{lrrrrr}
\hline \multirow{2}{*}{\multicolumn{1}{c}{ Denominación }} & \multicolumn{5}{c}{ Meses 2018 } \\
\cline { 2 - 6 } (+) Ventas & \multicolumn{1}{c}{ Enero } & Febrero & \multicolumn{1}{c}{ Marzo } & \multicolumn{1}{c}{ Abril } & \multicolumn{1}{c}{ Mayo } \\
\cline { 2 - 6 } (-) Costos de producción & $25.400,34$ & $26.053,85$ & $25.832,39$ & $25.178,87$ & $25.772,39$ \\
\hline (=) Utilidad bruta & $10.052,02$ & $9.888,70$ & $9.879,58$ & $9.688,00$ & $9.879,58$ \\
\hline (-) Gastos administrativos & $15.348,32$ & $16.165,15$ & $15.952,81$ & $15.490,87$ & $15.892,81$ \\
\hline (-) Gastos en ventas & 3172,47 & 3101,14 & 3093,94 & 3038,74 & 3086,74 \\
\hline (=) Utilidad operativa & 500,00 & 500,00 & 500,00 & 500,00 & 500,00 \\
\hline (-) Gastos financieros & 11675,85 & 12564,01 & 12358,87 & 11952,13 & 12306,07 \\
\hline (=) Utilidad antes de & 713,16 & 713,16 & 713,16 & 713,16 & 713,16 \\
impuestos & & & & & 11238,97 \\
\hline \\
(-) Impuestos 25\% & 10962,69 & 11850,85 & 11645,71 & 11592,91 \\
\hline (=) Utilidad neta & $2.740,67$ & $2.962,71$ & $2.911,43$ & $2.809,74$ & $2.898,23$ \\
\hline
\end{tabular}

Fuente: (Guallpa, 2018)

\section{Indicadores de rentabilidad del aserradero A2}

Las ventas de la fábrica por comercializar tablas y costeras de eucalipto generaron 61,49\% de utilidad bruta al aserradero A2 para el periodo de 5 meses del año 2018 (Tabla 9).

Tabla 9. Indicadores de rentabilidad del aserradero A2

\begin{tabular}{|c|c|}
\hline Denominación & Total \\
\hline (+) Ventas & $128.237,85$ \\
\hline (-) Costos de producción & $49.387,87$ \\
\hline (=) Utilidad bruta & $78.849,97$ \\
\hline (=) Utilidad neta & $42.968,34$ \\
\hline Margen Bruto Utilidad (MBU) & $61,49 \%$ \\
\hline Margen de Utilidad Neta (MUN) & $87,00 \%$ \\
\hline
\end{tabular}

El margen de utilidad neta, indica que por cada USD 100 que ingresan al negocio, después de pagar los costos (fijos, variables, gastos e impuestos) por aserrar madera de eucalipto, le sobra USD 87 al aserradero A2 (Tabla 9). 
Con los resultados de esta investigacion, se evidencia que el aserradero A2 con el uso del sistema sierra circular más sierra de cinta optimiza su proceso productivo al aumentar la productividad y garantizar la calidad de unidades de pie tablar de primera, y segunda de eucalipto, según sus niveles de venta durante el periodo enero a junio de 2018 (Salas \& Cortabarría, 2014). Cuya transacción de los tres productos de madera, generan utilidad bruta de $53,79 \%$, 61,49\% y como margen de utilidad neta 61 y 87 dólares respectivamente. Indicadores que permiten verificar, sí los planes de acción se están cumpliendo a cabalidad o generar alternativas de gestión sostenibles de las fábricas a fin de lograr su funcionamiento óptimo a mediano y largo plazo (Nava, 2009).

\section{Conclusiones}

- Los costos de producción por pie tablar de E. globulus son similares con valores de 0,29 y $0,27 \mathrm{USD} / \mathrm{pt}$ al trabajar con sierra circular versus el sistema sierra circular y de cinta respectivamente. Cuya optimización de la materia prima, se obtiene cuando se utiliza el sistema de aserrado combinado en el aserradero A2, mismo que influye con la disminución del 16,44\% de los costos de materia prima en relación al 44,28\% del aserradero A1.

- La rentabilidad de los dos proyectos de aserradero por vender tablas de primera, tablas de segunda y costeras de eucalipto generaron valores superiores al 50\% como margen de utilidad bruta para los aserraderos evaluados, siendo ideales para estos negocios, y en el caso del margen de utilidad neta del aserradero A2 por ser más alto con 26 USD, es el mejor en referencia al aserradero A1.

- Realizar estudios de mercado de otros productos derivados de la madera de $E$. globulus y de ciertos servicios que pueda ofertar cada aserradero de tal modo que se incorpore valor agregado, se genere más puestos de trabajo y asegurar la permanencia del negocio a mediano y largo plazo.

\section{Referencias bibliográficas.}

Añazco, M., Morales, M., Palacios, W., Vega., \& Cuesta, A. (2010). Sector Forestal Ecuatoriano: propuestas para una gestión forestal sostenible. Quito: Serie Investigación y Sistematización N8. Programa Regionl ECOBONAINTERCOOPERATION.

Coronel de Renolfi, M., Díaz, F., Cardona, G., \& Ruiz, P. (2012). Tiempos, rendimientos y costos del aserrado de Algarrobo blanco (Prosopis alba) en Santiago del Estero, Argentina. Quebracho. 20 (1,2): 15-28

Cubbage, F. W., Davis, R. R., \& Frey, G. E. (2011). Guía para la evaluación económica y financiera de proyectos forestales comunitarios en México.Documento de Trabajo 
Forestal Latinoamericano $\mathrm{N}^{\circ}$ 2. Washington, DC 20433 USA: Banco Mundial Región de Latioamérica y El Caribe.

De la Hoz Suárez, B., Ferrer, M., \& De la Hoz Suárez, A. (2008). Indicadores de rentabilidad: herramientas para la toma de decisiones financieras en hoteles de categoría media ubicados en Maracaibo. Revista de Ciencias de Sociales. 14 (1): 88-109.

Ecuador Forestal. (Julio de 2013). Organización Ecuador Forestal. http://ecuadorforestal.org/wp-content/uploads/2013/03/PE_Industrias.pdf

Fontalvo, T., De la Hoz Granadillo, E., \& Vergara, J. C. (2012). Aplicación de análisis discriminante para evaluar el mejoramiento de los indicadores financieros en las empresas del sector alimento de Barranquilla - Colombia. Ingeniare. Revista Chilena de ingeniería. 20 (3): 320-330.

García de la Figal, A. (2018). Teoría y Metodología de la Investigación Científica. La Habana, Cuba: Alfaomega

García, J. D., Morales, L., \& Valencia, S. (2001). Coeficientes de aserrío para cuatro aserraderos banda en el Sur de Jalisco. Foresta-AN. Nota técnica Núm. 5. UAAAN, Saltillo, Coah. 12 p.

Guallpa, M. (2018). Rendimiento de madera aserrada de Eucalyptus globulus Labill (Eucalipto) con sierra circular y de cinta en el Cantón Riobamba. Año 2018 (Tesis de maestría). Universidad Técnica Estatal de Quevedo, Quevedo, Ecuador.

Guallpa, M., Rosero, S., Montenegro, G., \& Quinchuela, D. (2018). Estimación de los residuos forestales en los aserraderos de tres cantones, Zona 3 interandina, Ecuador. European Scientific Journal, 14 (30), 228-239.

Guallpa, M., Suatunce, J., \& Canchignia, H. (2019). Tiempos y rendimiento en el proceso de aserrado de Eucalyptus globulus Labill, con sierra circular y de cinta. Enfoque UTE, 10 (2), 126-143.

Hidalgo, D. (2009). Análisis de la Situación de la Empresa: Ratios de Rentabilidad II. Actualidad Empresarial, No 176

Hernández, A. (2016). Determinación de costos en la industria del aserrío. Guia básica. México.

Jaramillo, F. (2009). ¿Cómo hacer análisis financiero?. Bogotá: Alfa omega.

López, N., \& Muñoz, J. (2017). La producción forestal una actividad con alto potencial en el Ecuador requiere un cambio de visión. Bosques Latitud Cero, 7 (1), 69-76. 
Ministerio del Ambiente del Ecuador. MAE. (2010). Aprovechamiento de los Recursos Forestales 2007-2009. Quito, Ecuador.

Ministerio del Ambiente de Ecuador. MAE. (2013). Sistema nacional de control forestal. Recuperado el 10 de octubre de 2016 de http://www.ambiente.gob.ec/wpcontent/uploads/downloads/2015/07/CONTROL-FORESTAL.pdf.

Maturana, S., Pizani, E., \& Vera, J. (2010). Scheduling production for a sawmill: A comparison of a mathematical model versus a heuristic. Computers \& Industrial Engineering, 59(4), 667-674.

Muñoz, M. (2010). Perfil de la factibilidad. Quito, Ecuador.

Nava, M. (2009). Análisis financiero: una herramienta clave para una gestión financiera eficiente. Revista Venezolana de Gerencia, 14 (48), 606-628.

Pacheco, J. (2015). Gestión de datos gráficos y tablas dinámicas con excel. Ecuador: Macro.

PRO ECUADOR. (2013). Muebles y productos de madera - Pro Ecuador. Recuperado de: http://www.proecuador.gob.ec/wp-content/uploads/2015/06/Perfiles-de-InversionesPromocion-de-Inversiones/Perfiles-de-Inversion/Muebles-y-Productos-deMadera.pdf

Salas, K., \& Cortabarría, L. (2014). Análisis competitivo del sector madera y muebles de la región Caribe de Colombia. Prospectiva, 12 (1), 79-89.

Secretaría Nacional de Planificación y Desarrollo, S. (2017). Plan Nacional de Desarrollo 2017-2021. Toda una Vida. SENPLADES. Quito.

Soto, J., Aguirre, J., Méndez, J., \& Páez, G. (2000). Evaluación económica y ambiental de residuos forestales en aserraderos de Costa Rica. Revista Forestal Centroamericana (CATIE), 30, 29-33.

\section{【L Ciencia}




\section{PARA CITAR EL ARTÍCULO INDEXADO.}

Guallpa Calva, M. Ángel, Arcos Torres, J. F., Suatunce Cunuhay, J. P., \& Canchignia Martínez, H. F. (2021). Evaluación financiera de la producción y venta de madera aserrada de Eucalyptus globulus Labill elaborada con sierra circular y de cinta. Visionario Digital, 5(1), 70-84.

https://doi.org/10.33262/visionariodigital.v5i1.1541

\section{DDigital}

El artículo que se publica es de exclusiva responsabilidad de los autores y no necesariamente reflejan el pensamiento de la Revista Visionario Digital.

El artículo queda en propiedad de la revista y, por tanto, su publicación parcial y/o total en otro medio tiene que ser autorizado por el director de la Revista Visionario Digital.
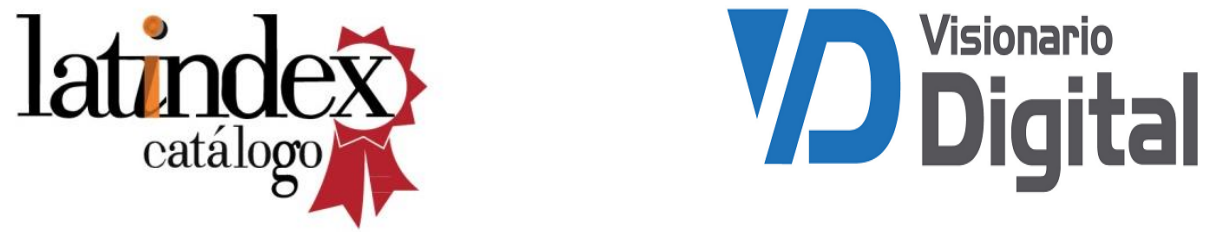